\title{
Mini-Review
}

\section{Role of Hyaluronan-Mediated CD44 Signaling in Head and Neck Squamous Cell Carcinoma Progression and Chemoresistance}

\author{
Steven J. Wang* and Lilly Y.W. Bourguignon ${ }^{\dagger}$ \\ From the Departments of Otolaryngology-Head and Neck \\ Surgery, ${ }^{*}$ and Medicine-Endocrine Unit, ${ }^{\dagger}$ Veterans Affairs \\ Medical Center, University of California at San Francisco, \\ San Francisco, California
}

Head and neck squamous cell carcinoma (HNSCC) is an aggressive malignancy that may involve the oral cavity, pharynx, larynx, and paranasal sinuses. The mechanisms of tumor progression underlying the clinical behavior of HNSCC remain unclear. CD44 comprises a family of transmembrane receptors that can give rise to multiple CD44 variant isoforms. Hyaluronan (HA), a major extracellular matrix component is the primary ligand for $\mathrm{CD} 44$ receptors. $\mathrm{HA}$ and CD44 signaling play an important role in HNSCC progression. Several CD44 variant isoforms (including v3-, v6-, and v10-containing isoforms) are associated with advanced disease, possibly through unique growth factor interactions with binding domains in the inserted variant regions of the cytoplasmic domain of CD44. In HNSCC, HA mediates the formation of a complex including CD44 and the epidermal growth factor receptor (EGFR) which is overexpressed in a large proportion of HNSCCs. Downstream effectors under EGFR regulation are activated, promoting promote cell growth and tumor survival. The leukemia-associated Rho-guanine nucleotide exchange factor (LARG) also associates with CD44 and EGFR to promote several Ras and RhoA pathway effectors, leading to cell migration, growth, and tumor survival. The secretion of matrix metalloproteinases, necessary for tumor cell invasion, is also regulated by these HA/CD44-mediated pathways. Finally, EGFR-mediated pathways play major roles in the HA/CD44 promotion of chemoresistance in HNSCC. Understanding HA/CD44-mediated signaling pathways may lead to improved treatment of HNSCC. (Am J Pathol 2011, 178: 956-963; DOI: 10.1016/j.ajpath.2010.11.077)
Head and neck squamous cell carcinoma (HNSCC) is a malignancy that may involve the oral cavity, pharynx, larynx, and paranasal sinuses. It is the sixth most common cancer worldwide. ${ }^{1}$ Advanced HNSCC is an aggressive disease, associated with major morbidity and mortality. The 3-year survival rate for patients with advanced-stage HNSCC treated with standard therapy is only $30 \%-50 \% .{ }^{1}$ Resistance to standard therapy continues to be a limiting factor in the treatment of HNSCC. Nearly $40 \%$ to $60 \%$ of HNSCC patients subsequently develop locoregional recurrences or distant metastases. There is a great need to clarify the mechanisms of tumor progression underlying the clinical behavior of HNSCC.

CD44 comprises a family of transmembrane receptors found on many different benign and malignant cells. The human gene (CD44) contains 19 exons. ${ }^{2}$ Up to 10 exons (primarily exons 6 through 14) may be alternatively spliced to give rise to multiple variant CD44 isoforms (eg, CD44 v3, CD44 v6, CD44 v10, etc) that, along with standard CD44 (CD44s; 85-kDa isoform with no variable exons), make up the CD44 class of receptors. Alternative splicing and post-translational modifications are tightly regulated and permit expression of multiple different CD44 isoforms. The many splicing possibilities of the variable exons of CD44 could in theory give rise to a vast number of CD44 variants, although relatively few have been described to date. Various CD44 variant isoforms are differentially expressed in normal and malignant cells, and confirmation of CD44 isoform expression in HNSCC is well documented, in both tissue specimens and established cell lines. ${ }^{3-6}$

Supported by Veterans Affairs (VA) Career Development Award (S.W.) VA Merit Review grant (L.Y.W.B.), and U.S. Public Health grants (R01 CA66163, R01 CA78633, and P01 AR39448 to L.Y.W.B.). L.Y.W.B. is a VA Senior Research Career Scientist.

Accepted for publication November 16, 2010.

Address reprint requests to Steven J. Wang, M.D., Department of Otolaryngology-Head and Neck Surgery, University of California, San Francisco, 2233 Post St, Box 1225, San Francisco, CA 94115. E-mail: swang@ohns.ucsf.edu. 
All isoforms of the CD44 membrane receptor share a common ligand-binding region for hyaluronan (HA), a glycosaminoglycan component of the extracellular matrix $(\mathrm{ECM}){ }^{2}$ Recently, HA has been studied with regard to its ability to promote various CD44-mediated signaling pathways. ${ }^{2,7}$ HA/CD44 signaling has been linked to solid tumor progression, including invasion, metastasis, and chemoresistance. ${ }^{7,8}$ In cancer cells, HA interaction with CD44 promotes multiple signaling pathways that influence tumor cell progression behaviors in a variety of solid tumors. Because all CD44 isoforms are capable of HA interaction, HA-mediated signaling may involve CD44s or variant isoforms.

An accumulating body of evidence indicates that $\mathrm{HA}$ and CD44 signaling play an important role in HNSCC tumor progression and chemoresistance. Here we review the recent scientific literature regarding the role of $\mathrm{HA}$ mediated CD44 signaling in HNSCC.

\section{CD44 Variant Isoform Expression Is Associated with HNSCC Progression}

All CD44 isoforms contain a conserved extracellular binding domain for $\mathrm{HA}$, along with common cytoplasmic domains capable of interacting with cytoskeleton proteins to activate cell signaling pathways. ${ }^{9}$ The ability of CD44 receptors to provide a direct link between the ECM and the cytoskeleton, coupled with their ability to interact with a multitude of signaling kinases, explains how one family of molecules is able to mediate diverse cellular functions.

CD44 isoforms are expressed to varying degrees in both normal and tumor cells, suggesting a normal cellular function for many of the different CD44 receptor isoforms. How the same CD44-mediated signaling pathways can promote both normal and malignant cellular behaviors could be explained by several different mechanisms. For example, the regulation of CD44 signaling includes variable $\mathrm{N}$ - or O-linked glycosylation of the CD44 extracellular domain that differentially modulates the interaction of cytoskeletal proteins such as ankyrin with the cytoplasmic domain. ${ }^{10}$ It is thought, however, that the most important mechanism regulating CD44 signaling is through alternative exon splicing leading to expression of CD44 variant isoforms. In cancer cells, the exon-splicing mechanism can result in overexpression or novel expression of certain CD44 variant isoforms. ${ }^{11,12}$ The insertion of variant exons into the extracellular component of the CD44 receptor has been shown to alter the signaling properties of the CD44 receptor by providing additional binding domains for molecules other than HA. ${ }^{11}$ The insertion of variant exons may also result in changes to the HAbinding affinity of the CD44 isoform, further altering its signaling behavior.

Overexpression of several CD44 variant isoforms has been associated with tumor progression, suggesting that these CD44 isoforms may have unique signaling properties. In colon cancer, CD44 v3 has been shown to promote invasion and resistance to apoptosis, and CD44 v6 has been associated with metastasis and shorter disease-free survival. ${ }^{13,14}$ In lung cancer, there is preferen- tial CD44 variant expression in squamous cell carcinoma and bronchioalveolar carcinoma, in which the v5 and v6 variants appear to promote metastasis. ${ }^{15,16}$ Numerous reports have shown that $\mathrm{CD} 44$ variants promote breast cancer progression, including the association of CD44 $\mathrm{v}$-containing isoforms and breast cancer metastasis. ${ }^{17}$

Because of evidence linking CD44 variant isoform expression with tumor progression in a variety of solid malignancies, multiple groups have studied the role of CD44 variant isoforms in HNSCC. Nonetheless, the role of CD44 variant isoforms in HNSCC remains controversial. The literature is conflicting regarding the significance of the under- or overexpression of CD44 variant isoforms in HNSCC.

Whereas some studies have found a correlation between increased CD44 variant expression and HNSCC progression, other studies have reported no correlation or negative correlation. Reategui et al ${ }^{18}$ described a novel CD44 v3 isoform in both tissue and soluble form that correlated with HNSCC status. Wang et $\mathrm{al}^{19,20}$ reported that CD44 v3-containing isoforms were associated with HNSCC lymph node metastasis and advanced T status, CD44 v6-containing isoforms were associated with perineural invasion and shorter survival, and CD44 $\mathrm{v} 10$-containing isoforms were associated with distant metastasis and failure of radiotherapy. Kawano et $\mathrm{al}^{3}$ found correlations between CD44 v6 expression in HNSCC and tumor volume, lymph node metastasis, and shorter survival. Others, however, have reported that down-regulation of various CD44 variant isoforms correlated with a worse prognosis. Kanke et al ${ }^{4}$ reported that down-regulation of CD44 v2 correlated with poorer differentiation and shorter overall survival, whereas down-regulation of CD44 v6 correlated with a higher rate of cervical metastasis. Other groups, including Van $\mathrm{Hal}$ et $\mathrm{al}^{6}$ and HeroldMende et al, ${ }^{5}$ found no correlation between CD44 splice variants and any clinicopathologic variables, and these authors concluded that CD44 variant isoforms do not play a role in HNSCC progression.

There are several possible explanations for these discrepant results. First, different studies have used different antibodies, making comparisons across reports from different research groups difficult. Certain CD44 variant domain epitopes may become hidden and not recognized by some antibodies because of posttranslational changes (eg, glycosylation) that alter the three-dimensional conformation of the protein. In addition, assessment of immunostaining positivity is dependent on what region of the tumor is examined, and it has been reported that there are often large areas within the tumor that do not stain for many CD44 isoforms. ${ }^{21-23}$ Tissue heterogeneity appears to be another important factor in the conflicting results, with the specific region of the tumor that is examined apparently having high importance in the determination of CD44 expression levels. CD44s and CD44 variant receptors appear to be concentrated at invasive fronts of HNSCC tumors, but have decreased expression in other parts of the tumor. ${ }^{21}$ It has been hypothesized that the major CD44 ligand HA, which is present at high levels in the ECM surrounding HNSCCs, is responsible for the stimulation of CD44 to 
promote migration, invasion, and metastasis. Hyaluronan has been reported to stimulate both CD44 and lymphatic vessel endothelial hyaluronan receptor (LYVE-1) to promote nodal metastasis in HNSCC. ${ }^{22}$

Although we acknowledge that the recent scientific literature is without broad consensus, support for a link between CD44 variant expression and HNSCC progression is bolstered by the recent description of three CD44 variants associated with HNSCC progression in both clinical specimens and in in vitro studies. ${ }^{20}$ Furthermore, the identification of CD44 as a putative cancer stem cell marker, as well as a marker for chemoresistance, is also consistent with the notion that certain CD44 variant isoforms may be contributory to HNSCC progression. ${ }^{8,24}$

\section{CD44 v3}

The mechanism of action for most CD44 variant isoforms in solid malignancies is not well understood. Several CD44 variant exons are known to contain binding domains for various growth factor ligands. The $v 3$ exon is known to contain important glycosaminoglycan (GAG) attachment sites, and it has been postulated that tumor cells with CD44 receptors exhibiting these GAG sequences are involved with heparin binding growth factors such as basic fibroblast growth factor, vascular endothelial growth factor (VEGF), and heparin binding epidermal growth factor. ${ }^{17}$ Thus, CD44 v3-containing isoforms appear to be capable of promoting tumor cell proliferation in an HA-independent manner, through the presence of additional growth factor binding sites. The CD44 v3 isoform is expressed in the metastatic breast tumor cell line Met-1, in which it has been shown to bind VEGF, suggesting that this isoform may promote breast tumor-associated angiogenesis. ${ }^{17,25}$ CD 44 v3 also appears to be linked to several other tumorigenic molecules, promoting tumor cell migration and the invasive tumor cell phenotype. In particular, CD44 v3 colocalizes with the active form of matrix metalloproteinase-9 (MMP-9) in Met-1 cells, promoting degradation of the ECM to facilitate tumor cell invasion. ${ }^{25}$ This isoform also up-regulates cytoskeleton function, through ankyrin, to activate the membrane-associated actomyosin contractile system and mediate tumor cell migration. ${ }^{25}$

The role of CD44 v3 isoforms in HNSCC progression is highlighted by studies showing an association of v3-containing isoforms with HNSCC growth, migration, and MMP expression. ${ }^{11,19,20}$ Transfection of a CD44 v3 isoform into a nonexpressing HNSCC cell line also resulted in significantly increased tumor cell migration, but not proliferation. ${ }^{18}$ Treatment of a CD44 v3 isoform expressing HNSCC cell line with anti-CD44 v3 antibody decreased in vitro proliferation and increased cisplatin sensitivity. $^{20}$ Using the same anti-CD44 v3 antibody, immunohistochemical tissue analysis revealed that CD44 v3 isoforms were preferentially expressed in metastatic lymph nodes. Additionally, strong CD44 v3 isoform expression in primary tumors was significantly associated with advanced T status and positive lymph nodes, but did not correlate with disease-free interval. ${ }^{20}$ In summary, there are several in vitro and histopathological studies suggesting that CD44 v3 isoforms are involved in HNSCC progression behaviors. To verify these findings, further research is needed to elucidate the mechanisms of CD44 v3 signaling.

\section{CD44 v6}

CD44 v6-containing isoforms also appear to promote tumor progression. Transfection of CD $44 \mathrm{v} 6$ converted nonmetastatic rat carcinoma cells into metastatic cells, and co-injection of anti-CD44 v6 antibody into these same cells suppressed their metastatic behavior. ${ }^{12,26}$ The CD44 v6 splice variant was also found to stimulate sustained increased mitogen activated protein kinase (MAPK) levels and subsequent downstream Ras signaling, resulting in increased tumor cell proliferation. ${ }^{12}$

Because of evidence linking CD44 v6 as a marker for HNSCC tumor proliferation and metastasis, a phase I clinical trial was conducted to evaluate anti-CD44 v6 antibody therapy for the treatment of incurable HNSCC, with a $10 \%$ response rate reported. ${ }^{23}$ Administration of anti-CD44 v6 antibody to a CD44 v6 isoform-expressing HNSCC cell line resulted in decreased cell proliferation and increased cisplatin sensitivity. ${ }^{20}$ Using the same anti-CD44 v6 antibody, immunohistochemical analysis of HNSCC tissue specimens indicated that CD44 v6 isoforms were preferentially expressed in metastatic lymph nodes, and strong expression of CD44 v6 in primary tumors was significantly associated with advanced $\mathrm{T}$ status, perineural invasion, and shorter disease-free survival. ${ }^{20}$ Although these studies are small, the association of CD44 v6 isoforms with clinically advanced HNSCC and the intriguing results of anti-CD44 v6 therapy in a phase I clinical trial suggest a need for further study of the role of this CD44 variant isoform in HNSCC.

\section{CD44 v10}

Although all CD44 isoforms share HA binding domains, certain CD44 variant isoforms, such as CD44 v10, exhibit significantly reduced affinity for $\mathrm{HA}$ binding. ${ }^{25}$ It is thought that the reduction in HA-mediated cell adhesion in tumor cells expressing CD44 v10 may be the earliest event in the onset of tumor migration and invasion. The unique structure of CD44 v10 may also cause constitutive activation of CD44-cytoskeleton interactions that induce tumor cell migration and invasion. CD44 v10-containing isoforms have been reported to promote tumor progression in breast and renal cell carcinoma. ${ }^{25,27}$ Studies have revealed that CD44 v10-transfected breast tumor cells display higher migration/invasion potential, produce higher levels of basic fibroblast growth factor and interleukin-8, and exhibit more potent tumor growth potential than parental control cells. ${ }^{25}$ Treatment of a CD44 v10 isoform-expressing HNSCC cell line with anti-CD44 v10 antibody resulted in decreased tumor cell proliferation and increased cisplatin sensitivity. ${ }^{20}$ The same anti-CD44 v10 antibody was used in the analysis of HNSCC clinical tissue specimens, demonstrating that CD44 v10 isoforms were preferentially expressed in metastatic lymph nodes. In addition, strong expression of CD44 v10 in primary 
tumors was significantly associated with distant metastasis, failure of radiotherapy, and shorter disease-free survival. ${ }^{20}$ Further studies to establish the interaction of the ligands involved in CD44 v10 signaling in HNSCC are needed to determine its role in HNSCC metastasis and treatment resistance.

\section{CD44 Variant Isoforms in Head and Neck Dysplastic Lesions}

Because there is evidence to support an association of CD44 and CD44 variant isoforms with HNSCC progression, it is natural to consider a putative role for CD44 receptors in head and neck dysplastic lesions. Increased CD44 and CD44 variant isoform expression is observed in most dysplastic lesions of the head and neck. ${ }^{21,28-31}$ Nonetheless, there are conflicting reports regarding CD44 status as a predictor of clinical outcomes in laryngeal and oral dysplasia. Osteopontin (a ligand with reported specificity for the CD44 v6 isoform) and CD44v6 are both overexpressed in laryngeal dysplasia and laryngeal SCC, and their levels correlate with degree of dysplasia, disease-free survival, and recurrence. ${ }^{31}$ Plasma osteopontin levels also correlate clinically with a worse response to chemoradiation treatment. ${ }^{32}$ CD44v6 expression was reported to correlate with developing both dysplastic and malignant oral epithelia. ${ }^{28}$ In contrast, loss of CD44 expression has been observed in adjacent nontumor epithelium in tongue cancer. ${ }^{29}$ Loss of CD44v7-8 was associated with worse prognosis in oral cavity dysplasias associated with betel quid chewing. ${ }^{30}$ Thus, regarding the role of CD44 variant isoforms in head and neck dysplasia, a few studies point to the importance of CD44 v6 and its ligand osteopontin in the development of dysplastic lesions and progression to HNSCC, but the role of other CD44 variant isoforms is uncertain.

\section{Interaction of HA and CD44 Promotes EGFR- Mediated Pathways to Increase HNSCC Growth, Survival, and Chemoresistance}

The epidermal growth factor receptor (EGFR) is expressed in nearly all HNSCCs, and its overexpression is associated with poor prognosis and diminished survival. Pharmaceutical agents targeting EGFR and EGFR signaling pathways have recently been introduced for the treatment of advanced HNSCC. ${ }^{33}$ The concept that EGFR and EGFR signaling might be linked to CD44 was first suggested for cervical carcinoma cell lines, and subsequently was demonstrated for breast cancer and glioblastoma cell lines. ${ }^{34-36}$ CD44 colocalized with EGFR in both cervical carcinoma clinical specimens and cell lines, and increased CD44 expression resulted in enhanced EGFR activity. ${ }^{34} \mathrm{CD} 44$ also colocalized with both EGFR and ERBB2 in metastasizing breast cancer cells. ${ }^{35}$ CD44 and EGFR interact in glioblastoma cells, and this interaction resulted in enhanced HA-mediated phosphorylation of extracellular signal regulated kinases 1 and 2 (ERK1 and ERK2) ${ }^{36}$ Additionally, co-expression of CD44 and EGFR in glioma cell lines differentially up-regulated the HA-induced expression of a number of genes associated with cellular invasion and proliferation, including TIMP metallopeptidase inhibitor 1 (TIMP1) and MYC (alias C-Myc). Because of the apparent importance of the EGFR pathway in HNSCC, our group investigated and reported a novel linkbetween HA-CD44 interaction and EGFR signaling in HNSCC. ${ }^{37,38}$

\section{CD44-EGFR-Mediated Oncogenic Signaling}

Analysis of anti-CD44-mediated immunoprecipitates from HNSCC cell lysates indicates that HA/CD44 interaction leads to CD44-EGFR complex formation. ${ }^{37,38}$ HA treatment was shown to promote phosphorylation of EGFR, activating the EGFR tyrosine kinase (TK) and promoting downstream EGFR-mediated pathways including Ras, RhoA, Rho kinase, and phosphatidylinositol-3 (PI-3) kinase signaling. ${ }^{37}$ The CD44-EGFR complex also associates with leukemia-associated Rho-guanine (LARG) nucleotide exchange factor to effect downstream signaling through ras and RhoA. Ras activation by HA-mediated CD44-LARG-EGFR complex formation promotes Raf-1 phosphorylation and MAPK activation. The stimulation of EGFR and downstream EGFR signaling activity by HA treatment was similar to epidermal growth factor stimulation. Wang et $\mathrm{al}^{38}$ reported that HA/CD44-mediated activation of EGFR signaling leads to MAPK phosphorylation/activation that results in increased HNSCC tumor cell growth; however, the HA-mediated increased cell growth was blocked by treatment with inhibitors of EGFR and MAPK.

One of the key downstream molecular effectors in EGFR-mediated cell survival is $\mathrm{PI}-3$ kinase. $\mathrm{Pl}-3$ kinase is capable of catalyzing the conversion of phosphatidylinositol-3,4-bisphosphate $\left(\mathrm{PIP}_{2}\right)$ to phosphatidylinositol 3,4,5-trisphosphate $\left(\mathrm{PIP}_{3}\right)$, which subsequently results in the activation of AKT. AKT has 3 subtypes: AKT-1, AKT-2, and AKT-3. HA/CD44 signaling in HNSCC activates all three AKT subtypes. Activated AKT down-regulates the cell's apoptotic potential, thus promoting increased proliferation and tumor cell survival. Torre et $\mathrm{al}^{39}$ reported that $\mathrm{HA} / \mathrm{CD} 44$ interaction increased PI-3 kinase activity in HNSCC cells; HA also promoted PI-3 kinase-mediated AKT phosphorylation, resulting in increased tumor cell survival.

\section{CD44-EGFR Signaling and Chemoresistance}

HA/CD44 signaling not only appears to promote EGFRmediated prosurvival effects, but also leads to increased chemoresistance in HNSCC through EGFR signaling pathways. Cisplatin is the most common anticancer drug used today for the treatment of HNSCC. Wang et $\mathrm{al}^{38,40}$ reported that $\mathrm{HA}$ can promote cisplatin resistance in several HNSCC cell lines; however, the HA-mediated cisplatin resistance could be abolished with inhibitors of EGFR and MAPK ${ }^{38}$ The mechanism of action of cisplatin is thought to involve apoptosis-induced cell death. Because AKT suppresses apoptosis, its activation by PI-3 kinase has been suggested to play a key role in cisplatin resistance. Torre et $\mathrm{al}^{39}$ demonstrated that LY-294002, 
an inhibitor of Pl-3 kinase, was capable of blocking HAmediated cisplatin resistance. Taken together, these reports suggest that HA/CD44 can promote cisplatin resistance in HNSCC through EGFR signaling pathways.

\section{Interaction of HA and CD44 Promotes Cytoskeleton Activation, Migration, Invasion, and Chemoresistance Pathways in HNSCC}

A hallmark of all solid malignancies is the ability to invade and/or metastasize to distant sites. Tumor cells possess altered signaling pathwaysthat lead to cytoskeleton activation and migration. Additionally, tumor cells secrete extracellular factors, such as matrix metalloproteinases, to allow breakdown and invasion of the surrounding ECM. Both $\mathrm{HA}$, which is a major component of the ECM, and its major ligand receptor, CD44, have been studied for their role in promoting tumor progression properties, including migration and invasion. The mechanisms of how HA and CD44 interact to promote cytoskeleton activation, migration, and invasion were initially elucidated in several cancer and noncancer models. $9,10,41-44$

RhoA signaling appears to be a critical pathway through which HA/CD44 interaction mediates cytoskeleton activation. One of several important mechanisms used by RhoA in the regulation of cellular functions is through alteration of intracellular $\mathrm{Ca}^{2+}$ levels. Phospholipase $\mathrm{C}(\mathrm{PLC})$ is a key mediator in intracellular $\mathrm{Ca}^{2+}$ mobilization. When activated by RhoA, PLCs first hydrolyze $\mathrm{PIP}_{2}$ into inositol trisphosphate $\left(\mathrm{IP}_{3}\right)$, resulting in $\mathrm{Ca}^{2+}$ release from intracellular stores. This $\mathrm{Ca}^{2+}$ release promotes various cell functions, including cytoskeleton activation, cell cycle progression, and proliferation. Another key RhoA pathway effector is Rho kinase (ROK), which has been shown to regulate several cytoskeletal proteins (such as myosin light chain phosphatase) that are highly involved in tumor migration and to promote the secretion of MMPs, which degrade the ECM during tumor invasion. 9, 10,39

HA/CD44 interaction has been shown to be tightly coupled with intracellular $\mathrm{Ca}^{2+}$ mobilization pathways and with RhoA pathways in many different cells. ${ }^{9,10,39}$ CD44 and RhoA are physically associated in metastatic breast cancer cells, and ROK was found to play a key role in CD44-ankyrin interaction and in RhoA-mediated breast cancer oncogenic signaling. ${ }^{41}$ In endothelial cells, HA treatment promoted CD44 interaction with ROK, leading to $\mathrm{IP}_{3}$ receptor-mediated $\mathrm{Ca}^{2+}$ mobilization and migration. ${ }^{9}$ In keratinocytes, HA-mediated $\mathrm{Ca}^{2+}$ mobilization promoted cortactin-cytoskeleton function, leading to adhesion and differentiation. ${ }^{44}$ CD44 is linked to ankyrin and has been shown to activate MMP-9 during active migration processes in metastatic breast cancer cells. ${ }^{10}$

\section{HAVCD44 Interaction with Cytoskeletal Proteins, Ankyrin, and ERM}

Ankyrin is a membrane-associated cytoskeletal protein that directly binds CD44. ${ }^{10}$ This CD44-ankyrin interaction causes cytoskeleton activation. Hyaluronan binding to
CD44 promotes colocalization of CD44 and ankyrin in cholesterol-containing lipid rafts, and this colocalization appears to be a key mechanism in regulating HA-mediated cytoskeleton function and tumor cell-specific behaviors (eg, cell survival, growth, and migration). Ankyrinand CD44-containing lipid rafts have been documented in three different tumor cell lines (breast cancer, ovarian cancer, and HNSCC). ${ }^{10}$

Up-regulated expression of the ezrin-radixin-moesin (ERM) family of cytoskeletal proteins is seen in HNSCC and is associated with poor prognosis. ${ }^{45}$ ERM proteins are linkers between membrane molecules such as CD44 and the cytoskeleton. ${ }^{45,46}$ Ezrin is a key regulator of tumor metastasis. CD44 interacts with ERM proteins and with merlin, a related protein. Loss of merlin results in increased HA/CD44-mediated tumorigenesis, and overexpression of merlin diminishes tumor cell growth. ${ }^{47}$ In HNSCC, moesin appears to interact with CD44 to degrade the ECM at the invasive front of oral cancers. ${ }^{48}$ Both the level of expression and the subcellular localization (cytoplasmic) of ERM proteins are indicators of clinical outcome in HNSCC. Higher expression and cytoplasmic localization of ERM proteins are indicators of poorer survival in HNSCC. ${ }^{45}$ Osteopontin, a ligand for CD44, colocalizes with CD44 and ezrin in fibroblasts, metastatic breast cancer cells, and HNSCC. ${ }^{46}$

\section{RhoA-Regulated PLC, ROK, and $\mathrm{Ca}^{2+}$ Signaling and Cytoskeleton Activation}

Similar mechanisms for CD44-mediated $\mathrm{Ca}^{2+}$ mobilization pathways to promote tumor migration appear to exist in HNSCC. In HNSCC, Bourguignon et a ${ }^{37}$ reported that CD44 physically associates in a multimolecular complex with LARG and EGFR. HA/CD44 interaction induces LARG-specific RhoA signaling. LARG molecules isolated from HNSCC cells were found to function as a GDP/GTP exchange factor for RhoGTPases, and the basal rate of bound GTP increased at least 2.4-fold with the addition of $\mathrm{HA}$.

To establish a linkage between HA/CD44-mediated LARG-RhoA signaling and intracellular $\mathrm{Ca}^{2+}$ regulation, one member of the PLC family, $\mathrm{PLC} \varepsilon$, was isolated from HNSCC cells with GDP- or GTP-loaded forms of RhoAGST-conjugated beads. PLC $\varepsilon$-RhoA interaction was found to be GTP-dependent. LARG-activated RhoA was shown to stimulate both the $\mathrm{PLC} \varepsilon$-mediated $\mathrm{IP}_{3}$ production and the $\mathrm{IP}_{3}$ receptor-triggered intracellular $\mathrm{Ca}^{2+}$ mobilization in HNSCC cells. HNSCC cells incubated with Fura-2/AM were treated with HA with or without pretreatment with inhibitors of PLC and $\mathrm{IP}_{3}$ receptor; fluorescence spectrophotometry demonstrated a rise in intracellular $\mathrm{Ca}^{2+}$ with $\mathrm{HA}$ treatment, but not in the presence of pretreatment with various inhibitors. ${ }^{40}$ These findings suggest that $\mathrm{Ca}^{2+}$ signaling in HNSCC cells involves both HA/CD44-dependent and RhoA/PLC/IP 3 receptorregulated processes.

Hyaluronan-stimulated intracellular $\mathrm{Ca}^{2+}$ mobilization mediates important components of the CD44 signaling pathways. ${ }^{9,43,44}$ Some of these $\mathrm{Ca}^{2+}$-mediated pathways 


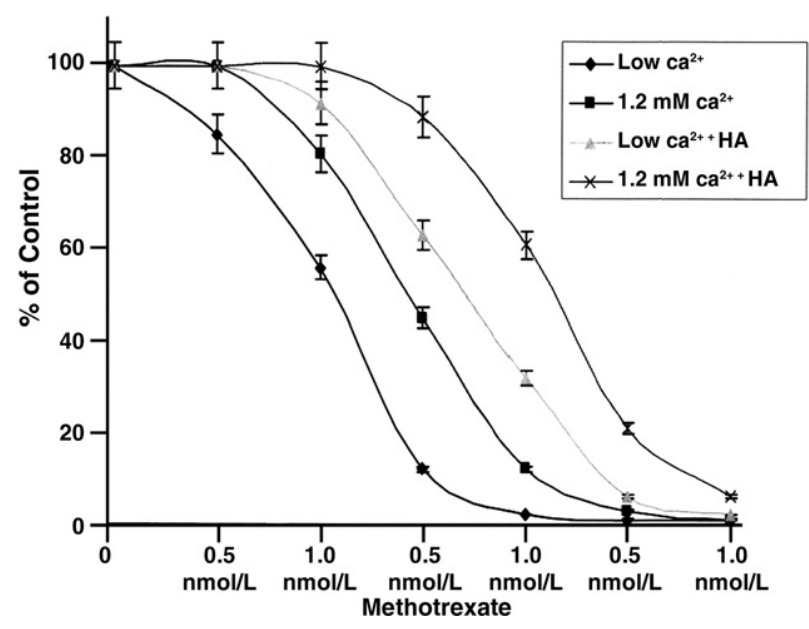

Figure 1. Analysis of $\mathrm{Ca}^{2+}$ concentration and methotrexate sensitivity. The head and neck squamous cell carcinoma cell line SCC4 was grown in serum-free medium containing either lowor $1.2 \mathrm{mmol} / \mathrm{L} \mathrm{Ca}^{2+}$ and was treated with increasing concentrations of methotrexate in the presence or absence of hyaluronan (HA) $(50 \mu \mathrm{g} / \mathrm{ml})$. An MTT assay was performed. Error bars represent calculated standard deviation.

may be mediated by the $\mathrm{Ca}^{2+}$ binding protein calmodulin. It is known that calmodulin is involved in the activation of several important enzymes, including calcium/calmodulin-dependent protein kinase type II (CaMKII), a ubiquitous serine/threonine protein kinase. In HNSCC cells, CaMKII activation by HA/CD44-mediated $\mathrm{Ca}^{2+}$ mobilization results in the phosphorylation of diverse substrates that promote various cell functions, including motility, cell cycle progression, and proliferation. ${ }^{37}$ CaMKII phosphorylates the cytoskeletal protein, filamin. These HA/CD44mediated effects on CaMKII and filamin lead to cytoskeleton reorganization and promote tumor cell migration.
Wang et $\mathrm{al}^{49}$ linked HA/CD44-dependent CaMKII activity to topoisomerase II regulation in HNSCC cells. Topoisomerase II is a critical regulator of DNA topology and function. Hyaluronan treatment promoted CaMKII-dependent topoisomerase II phosphorylation, resulting in enhancement of topoisomerase II activity and decreased cytotoxicity of etoposide (a topoisomerase II poison). These HA/ CD44-mediated effects on CaMKII and topoisomerase II activity enhanced tumor cell survival.

Another important effector of RhoA pathway signaling is Rho kinase (ROK). Activated ROK is known to phosphorylate a number of cytoskeletal proteins, such as myosin phosphatase and adducin, that are highly involved in tumor migration and to promote the secretion of MMPs involved in tumor invasion. Torre et $\mathrm{al}^{39}$ recently showed that HA/CD44 interaction increased ROK activity in HNSCC cells. Hyaluronan also promoted Rho kinase-mediated myosin phosphatase phosphorylation, resulting in enhanced tumor cell migration, and it increased activated MMP-2 and MMP-9 secretion.

\section{RhoAvCa ${ }^{2+}$ Signaling-Regulated Chemoresistance}

HA/CD44 interaction has been shown to promote resistance to multiple chemotherapeutic agents in HNSCC, including cisplatin, methotrexate, doxorubicin (Adriamycin), and etoposide. ${ }^{38,40,49}$ Several cell signaling mechanisms appear to promote CD44-mediated chemoresistance in HNSCC, including EGFR-related signaling pathways (as already described here). ${ }^{38}$ Recent work by our groupsuggests that regulation of $\mathrm{Ca}^{2+}$ may also affect chemoresistance (unpublished data). The median inhibitory concentration $\mathrm{IC}_{50}$ for the chemotherapy agent methotrexate in the HNSCC cell line SCC4 is dependent

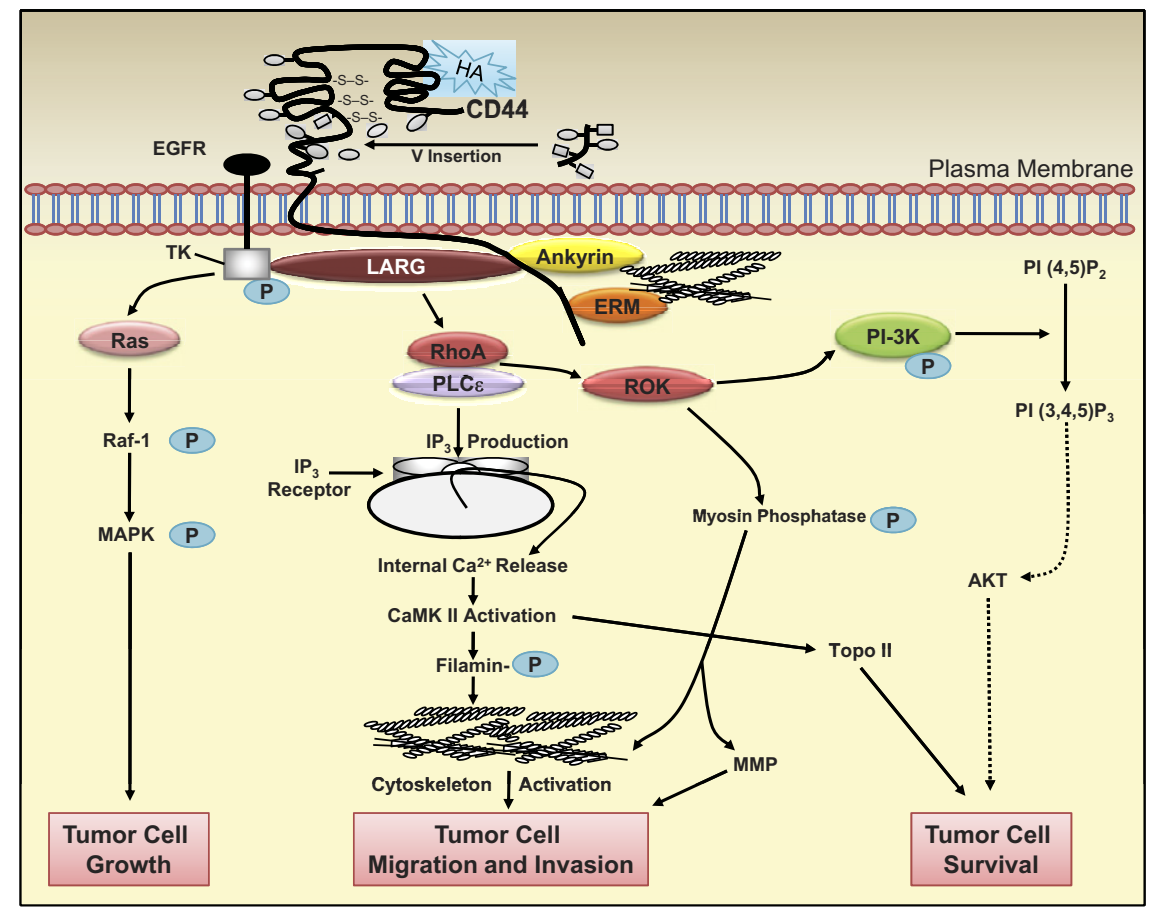

Figure 2. Our proposed model of hyaluronan (HA)-mediated CD44 signaling as promoting progression and chemoresistance in head and neck squamous cell carcinoma. In the transmembrane receptor $\mathrm{CD} 44$, a number of variant domains can be inserted into the extracellular portion of the receptor. After HA-CD44 interaction recruits and forms a CD44-EGFR-LARG multimolecular complex, multiple downstream signaling pathways are activated; cross-talk among Ras, RhoA, ROK, and PI-3 kinase can occur, promoting diverse head and neck cancer progression behaviors and chemoresistance. 
on both the $\mathrm{Ca}^{2+}$ level and the presence of $\mathrm{HA}$ (Figure 1). SCC4 cells grown in $1.2 \mathrm{mmol} / \mathrm{L} \mathrm{Ca}^{2+}$ medium had greater resistance to methotrexate than cells grown in low- $\mathrm{Ca}^{2+}$ medium, and the $\mathrm{IC}_{50}$ was increased in the presence of $\mathrm{HA}$ at both $\mathrm{Ca}^{2+}$ concentrations.

Phospholipase $\mathrm{C}$ and RhoA signaling, which mediate intracellular $\mathrm{Ca}^{2+}$ levels, has been shown to play roles in mediating chemoresistance in HNSCC. Wang et $\mathrm{al}^{38,40}$ demonstrated that HA-mediated cisplatin, methotrexate, and doxorubicin resistance could be eliminated with inhibition of PLC. Torre et $\mathrm{al}^{39}$ found that combined ROK and PI-3 kinase inhibition resulted in a synergistic prosurvival effect in the presence of cisplatin. Thus, HA-mediated chemoresistance in HNSCC may involve multiple pathways, including RhoA-mediated $\mathrm{Ca}^{2+}$ signaling.

\section{In Vivo Modeling of HA/CD44 Signaling in HNSCC}

Thorough review of the recent literature yields very little in terms of in vivo validation of mechanisms of CD44 signaling in HNSCC. This represents a deficiency in the current state of scientific knowledge. Most work to date related to CD44 signaling in HNSCC has involved analysis of in vitro data from HNSCC cell lines or relies on inferences from immunohistochemical analysis of patient tissue specimens. For breast cancer, there are in vivo data on CD44 signaling. Co-expression of CD44 v10 and $\mathrm{CD} 44 \mathrm{~s}$ through transfection of nonmalignant human breast epithelial cells was shown to promote tumorigenesis in athymic nude mice, but not for nontransfected or vector-only transfected parental cells. ${ }^{50}$ Furthermore, we can infer the importance of CD44 on HNSCC progression in vivo from studies of CD44 as a cancer stem cell marker in HNSCC, in which CD44-positive enriched HNSCC cells had greater tumorigenicity in nude mice, compared with CD44-negative HNSCC cells. ${ }^{24}$ Nonetheless, studies of CD44-mediated migration, metastasis, and chemoresistance or radiation resistance in an in vivo model of HNSCC are currently lacking in the literature.

\section{Conclusion}

In summary, an accumulating body of evidence highlights the important role of $\mathrm{HA}$ and CD44 signaling in HNSCC progression. In Figure 2 we present a model summarizing our current understanding of the role of $\mathrm{HA}$ and CD44 interaction with oncogenic signaling pathways to promote tumor progression and chemoresistance in HNSCC. Subsequent to the HA/CD44 interaction that recruits and forms a CD44-EGFR-LARG multimolecular complex, multiple downstream signaling pathways are activated, and cross-talk among Ras, RhoA, ROK, and $\mathrm{Pl}-3$ kinase can occur, further promoting diverse tumor progression behaviors. Understanding HA/CD44-mediated signaling pathways may lead to improved treatment, early detection, and prevention for this deadly disease. Research to date suggests that targeted inhibition of HA/CD44-mediated signaling combined with conventional chemotherapy agents may be an efficacious strat- egy, one that should be pursued to improve the future treatment of advanced HNSCC.

\section{References}

1. Jemal A, Siegel R, Ward E, Hao Y, Xu J, Thun MJ: Cancer statistics, 2009. CA Cancer J Clin 2009, 59:225-249

2. Turley EA, Noble PW, Bourguignon LY: Signaling properties of hyaluronan receptors. J Biol Chem 2002, 277:4589-4592

3. Kawano T, Nakamura Y, Yanoma S, Kubota A, Furukawa M, Miyagi Y, Tsukuda M: Expression of E-cadherin, and CD44s and CD44v6 and its association with prognosis in head and neck cancer. Auris Nasus Larynx 2004, 31:35-41

4. Kanke M, Fujii M, Kameyama K, Kanzaki J, Tokumaru Y, Imanishi Y, Tomita T, Matsumura Y: Clinicopathological significance of expression of CD44 variants in head and neck squamous cell carcinoma. Jpn J Cancer Res 2000, 91:410-415

5. Herold-Mende C, Seiter S, Born Al, Patzelt E, Schupp M, Zöller J Bosch FX, Zöller M: Expression of CD44 splice variants in squamous epithelia and squamous cell carcinomas of the head and neck. J Pathol 1996, 179:66-73

6. Van Hal NL, van Dongen GA, Stigter-Van Walsum M, Snow GB, Brakenhoff $\mathrm{RH}$ : Characterization of CD44v6 isoforms in head-andneck squamous-cell carcinoma. Int J Cancer 1999, 82:837-845

7. Toole BP: Hyaluronan-CD44 interactions in cancer: paradoxes and possibilities. Clin Cancer Res 2009, 15:7462-7468

8. Toole BP, Slomiany MG: Hyaluronan: a constitutive regulator of chemoresistance and malignancy in cancer cells. Semin Cancer Biol 2008, 18:244-250

9. Singleton PA, Bourguignon LY: CD44V10 interaction with Rho-kinase (ROK) activates inositol 1,4,5-triphosphate (IP3) receptor-mediated $\mathrm{Ca}^{2+}$ signaling during hyaluronan (HA)-induced endothelial cell migration. Cell Motil Cytoskeleton 2002, 53:293-316

10. Bourguignon LY: Hyaluronan-mediated CD44 activation of RhoGTPase signaling and cytoskeleton function promotes tumor progression. Semin Cancer Biol 2008, 18:251-259

11. Franzmann E, Weed DT, Civantos F, Goodwin WJ, Bourguignon LY: A novel CD44 v3 isoform is involved in head and neck squamous cell carcinoma progression. Otolaryngol Head Neck Surg 2001, 124:426-432

12. Günthert U, Hofmann M, Rudy W, Reber S, Zöller M, Haussmann I, Matzku S, Wenzel A, Ponta H, Herrlich P: A new variant of glycoprotein CD44 confers metastatic potential to rat carcinoma cells. Cell 1991, 65:13-24

13. Kuniyasu H, Oue N, Tsutsumi M, Tahara E, Yasui W: Heparan sulfate enhances invasion by human colon carcinoma cell lines through expression of CD44 variant exon 3. Clin Cancer Res 2001 7:4067-4072

14. Kuhn $S$, Koch $M$, Nübel $T$, Ladwein $M$, Antolovic $D$, Klingbeil $P$, Hildebrand D, Moldenhauer G, Langbein L, Franke WW, Weitz J, Zöller M: A complex of EpCAM, claudin-7, CD44 variant isoforms, and tetraspanins promotes colorectal cancer progression. Mol Cancer Res 2007, 5:553-567

15. Pirinen R, Hirvikoski P, Böhm J, Kellokoski J, Moisio K, Virén M, Johansson R, Hollmén S, Kosma VM: Reduced expression of CD44V3 variant isoform is associated with unfavorable outcome in non-small cell lung carcinoma. Hum Pathol 2000, 31:1088-1095

16. Mizera-Nyczak E, Dyszkiewicz W, Heider KH, Zeromski J: Isoform expression of CD44 adhesion molecules, $\mathrm{Bcl}-2$, p53 and $\mathrm{Ki}-67$ proteins in lung cancer. Tumour Biol 2001, 22:45-53

17. Kalish ED, lida N, Moffat FL, Bourguignon LY: A new CD44V3-containing isoform is involved in tumor cell growth and migration during human breast carcinoma progression. Front Biosci 1999, 4:A1-A8

18. Reategui EP, de Mayolo AA, Das PM, Astor FC, Singal R, Hamilton KL, Goodwin WJ, Carraway KL, Franzmann EJ: Characterization of CD44v3-containing isoforms in head and neck cancer. Cancer Biol Ther 2006, 5:1163-1168

19. Wang SJ, Wreesmann VB, Bourguignon LY: Association of CD44 V3 containing isoforms in tumor cell growth, migration, matrix metalloproteinase expression, and lymph node metastasis in head and neck cancer. Head Neck 2007, 29:550-558 
20. Wang SJ, Wong G, de Heer AM, Xia W, Bourguignon LY: CD44 variant isoforms in head and neck squamous cell carcinoma progression. Laryngoscope 2009, 119:1518-1530

21. Bánkfalvi A, Krassort M, Buchwalow IB, Végh A, Felszeghy E, Piffkó J: Gains and losses of adhesion molecules (CD44, E-cadherin, and beta-catenin) during oral carcinogenesis and tumour progression. J Pathol 2002, 198:343-351

22. Maula SM, Luukkaa M, Grénman R, Jackson D, Jalkanen S, Ristamäki R: Intratumoral lymphatics are essential for the metastatic spread and prognosis in squamous cell carcinomas of the head and neck region. Cancer Res 2003, 63:1920-1926

23. Orian-Rousseau V: CD44, a therapeutic target for metastasising tumours. Eur J Cancer 2010, 46:1271-1277

24. Prince ME, Sivanandan R, Kaczorowski A, Wolf GT, Kaplan MJ, Dalerba P. Weissman IL, Clarke MF, Ailles LE: Identification of a subpopulation of cells with cancer stem cell properties in head and neck squamous cell carcinoma. Proc Natl Acad Sci USA 2007, 104: 973-978

25. Bourguignon LY, Gunja-Smith Z, lida N, Zhu HB, Young LJ, Muller WJ, Cardiff RD: CD44v(3,8-10) is involved in cytoskeleton-mediated tumor cell migration and matrix metalloproteinase (MMP-9) association in metastatic breast cancer cells. J Cell Physiol 1998, 176:206-215

26. Seiter S, Arch R, Reber S, Komitowski D, Hofmann M, Ponta H, Herrlich P, Matzku S, Zöller M: Prevention of tumor metastasis formation by anti-variant CD44. J Exp Med 1993, 177:443-455

27. Li N, Tsuji M, Kanda K, Murakami Y, Kanayama H, Kagawa S: Analysis of CD44 isoform $v 10$ expression and its prognostic value in renal cell carcinoma. BJU Int 2000, 85:514-518

28. Rautava J, Soukka T, Inki P, Leimola-Virtanen R, Saloniemi I, Happonen RP, Heikinheimo K: CD44v6 in developing, dysplastic and malignant oral epithelia. Oral Oncol 2003, 39:373-379

29. González-Moles MA, Bravo M, Ruiz-Avila I, Esteban F, BasconesMartínez A, González-Moles S: Adhesion molecule CD44 expression in non-tumour epithelium adjacent to tongue cancer. Oral Oncol 2004, 40:281-286

30. Kuo MY, Cheng SJ, Chen HM, Kok SH, Hahn LJ, Chiang CP: Expression of CD44s, CD44v5, CD44v6 and CD44v7-8 in betel quid chewing-associated oral premalignant lesions and squamous cell carcinomas in Taiwan. J Oral Pathol Med 1998, 27:428-433

31. Staibano S, Merolla F, Testa D, lovine R, Mascolo M, Guarino V, Castellone MD, Di Benedetto M, Galli V, Motta S, Melillo RM, De Rosa G, Santoro M, Celetti A: OPN/CD44v6 overexpression in laryngeal dysplasia and correlation with clinical outcome. Br J Cancer 2007, 97:1545-1551

32. Snitcovsky I, Leitão GM, Pasini FS, Brunialti KC, Mangone FR, Maistro S, de Castro G Jr, Villar RC, Federico MH: Plasma osteopontin levels in patients with head and neck cancer undergoing chemoradiotherapy. Arch Otolaryngol Head Neck Surg 2009, 135:807-811

33. Ford AC, Grandis JR: Targeting epidermal growth factor receptor in head and neck cancer. Head Neck 2003, 25:67-73

34. Wobus M, Kuns R, Wolf C, Horn LC, Köhler U, Sheyn I, Werness BA, Sherman LS: CD44 mediates constitutive type I receptor signaling in cervical carcinoma cells. Gynecol Oncol 2001, 83:227-234

35. Wobus M, Rangwala R, Sheyn I, Hennigan R, Coila B, Lower EE, Yassin RS, Sherman LS: CD44 associates with EGFR and erbB2 in metastasizing mammary carcinoma cells. Appl Immunohistochem Mol Morphol 2002, 10:34-39

36. Tsatas D, Kanagasundaram V, Kaye A, Novak U: EGF receptor modifies cellular responses to hyaluronan in glioblastoma cell lines. J Clin Neurosci 2002, 9:282-288
37. Bourguignon LY, Gilad E, Brightman A, Diedrich, Singleton P: Hyaluronan-CD44 interaction with leukemia-associated RhoGEF and epidermal growth factor receptor promotes Rho/Ras co-activation, phospholipase $\mathrm{C} \varepsilon-\mathrm{Ca}^{2+}$ signaling, and cytoskeleton modification in head and neck squamous cell carcinoma cells. J Biol Chem 2006, 281: $14026-14040$

38. Wang SJ, Bourguignon LY: Hyaluronan and the interaction between CD44 and epidermal growth factor receptor in oncogenic signaling and chemotherapy resistance in head and neck cancer. Arch Otolaryngol Head Neck Surg 2006, 132:771-778

39. Torre C, Wang SJ, Xia W, Bourguignon LY: Reduction of hyaluronanCD44 mediated growth, migration, and cisplatin resistance in head and neck cancer due to inhibition of Rho-kinase and PI-3 kinase signaling. Arch Otolaryngol Head Neck Surg 2010, 136:493-501

40. Wang SJ, Bourguignon LY: Hyaluronan-CD44 promotes phospholipase $\mathrm{C}$-mediated $\mathrm{Ca}^{2+}$ signaling and cisplatin resistance in head and neck cancer. Arch Otolaryngol Head Neck Surg 2006, 132 : 19-24

41. Bourguignon LY, Zhu H, Shao L, Chen YW: CD44 interaction with Tiam1 promotes Rac1 signaling and hyaluronic acid-mediated breast tumor cell migration. J Biol Chem 2000, 275:1829-1838

42. Bourguignon LY, Zhu H, Shao L, Chen YW: CD44 interaction with c-Src kinase promotes cortactin-mediated cytoskeleton function and hyaluronic acid-dependent ovarian tumor cell migration. J Biol Chem 2001, 276:7327-7336

43. Singleton PA, Bourguignon LY: CD44 interaction with ankyrin and IP3 receptor in lipid rafts promotes hyaluronan-mediated $\mathrm{Ca}^{2+}$ signaling leading to nitric oxide production and endothelial cell adhesion and proliferation. Exp Cell Res 2004, 295:102-118

44. Bourguignon LY, Singleton PA, Diedrich F: Hyaluronan-CD44 interaction with Rac1-dependent protein kinase $\mathrm{N}$-gamma promotes phospholipase Cgamma1 activation. $\mathrm{Ca}(2+)$ signaling, and cortactin-cytoskeleton function leading to keratinocyte adhesion and differentiation. J Biol Chem 2004, 279:29654-29669

45. Madan R, Brandwein-Gensler M, Schlecht NF, Elias K, Gorbovitsky E, Belbin TJ, Mahmood R, Breining D, Qian H, Childs G, Locker J, Smith R, Haigentz M Jr, Gunn-Moore F, Prystowsky MB: Differential tissue and subcellular expression of ERM proteins in normal and malignant tissues: cytoplasmic ezrin expression has prognostic significance for head and neck squamous cell carcinoma. Head Neck 2006, 28 : 1018-1027

46. Martin TA, Harrison G, Mansel RE, Jiang WG: The role of the CD44/ ezrin complex in cancer metastasis. Crit Rev Oncol Hematol 2003, 46:165-186

47. Bai Y, Liu YJ, Wang H, Xu Y, Stamenkovic I, Yu Q: Inhibition of the hyaluronan-CD44 interaction by merlin contributes to the tumor-suppressor activity of merlin. Oncogene 2007, 26:836-850

48. Kobayashi H, Sagara J, Kurita H, Morifuji M, Ohishi M, Kurashina K, Taniguchi S: Clinical significance of cellular distribution of moesin in patients with oral squamous cell carcinoma. Clin Cancer Res 2004, 10:572-580

49. Wang SJ, Peyrollier K, Bourguignon LY: The influence of hyaluronanCD44 interaction on topoisomerase II activity and etoposide cytotoxicity in head and neck cancer. Arch Otolaryngol Head Neck Surg 2007, 133:281-288

50. lida N, Bourguignon LY: Coexpression of CD44 variant (v10/ex14) and CD44S in human mammary epithelial cells promotes tumorigenesis. J Cell Physiol 1997, 171:152-160 